\title{
[ 193 ]
}

\section{X.}

Aufgefundene chemi/che Natur einiger dem Pflanzen reiche und dem Thierreiche angehörenden Säuren,

₹ $\mathbf{0} \mathbf{m}$

Hofrath Döвereiner in Jena;

(eine Fortfetzung feiner phyfikalifch-chemifchen Bemerkungen in dief. Ann. J. I821 St. 3 S. 331 and St. 5 S. 84, ausgezogen aus mehreren Briefen an Gilbert.")

i. Verwandlung von Alkohol in EMfigure mittelit Edm. Davy's neuem fogen. Knall-Platin, und Folgerungen daraus.

Jena d. 30 Auguft 182 r.

Das von dem Hrin Edmund Davy vor kurzem befchriebene neue fogenannte Knall-Platin, ift nichts anders als das Suboxyd des Platins **).

) Die lange Verfpätung des Abdrucks diefer intereffanten Mit: theilungen ift zufatlig nicht ohne Vortheil; einzelne Notizen bleiben häufig unbeachtet, und gerade jetzt fangen Chemiker des Auslandes an, auf diefe Arbeiten des Hrn Prof. Dobbereiner aufmerkfam zu werden und fie zu wiederholen. Gilb.

") Hr. Ed mu und Da y y, Prof. dor Chemio zu Kork in Irland, hat diefes Präparat, das er durch Kochen von fchwefelfaurem Platin in Alkohol und nachmaligem Digeriren des Pulvers in Ammoniak erhielt, im Jahr 1820 in den Schriften der königl. Gerellfchaft der Wiffenfch, zu London bokannt gemacht. Befeuchtet mit Alkohol zerfetzt fich diefes Knall-Platiti augenblicklich, und entbindet dabei fo viel Hitze, dafo ein Antial. d. Pligfik: B. 72. Sti 2. J.1822. Si. 16. 


\section{[ 194$]$}

Ich hahe daflethe dargeftellt, und finde, dafs cs mit dem Alkohol eine höch/t merkwürdige electrifche Combination bildet, cine Kette, in welcher das Suboxyd die Funclion des Silbers oder Kupfers, und der Alkohol dic Stelle des Links behauptet. Letzterer wird dadurch fo ausgezeichnet pofitiv electrifch, dafs er Schnell' und in grofser Menge Sauerftoffgas abforbirl, und entweder glïhend verbrennt, oder in Effig/äure iibergeht. Das erftere findet ftatt, wenn der Alkolol als Dampf in atmof pharifcher Luft veibreitet mit dem trocknen Platin-Snboxyd in Berührung kommt; das letztere, weni man den Alkolıol im tropfbar-flülfigen Zuftande mit dem Suboxyde in Berührung bringt, unter einer mit atmofphärifcher Lutt oder mit Sauerftoffgas gefüllten Glocke. Im letzien Falle beginnt die Thütigkeit nit Abforplion von Sauerfoffgas; daun wird Warme frei, der Alkoliol füngt an zu verdampfen und verwandelt fich unter fortdauernd vermehrter Verzehrung von Sauerftoffgas, erft in eine dem von mir befchriebenen Sanerfto(f-Aether *) analoge Subftanz, und hieranf, wenn noch Sauerfioffgas vorhanden ift, in Effigfäure und Waffer. Kohlenläure wird dabei nicht gebildet.

Glühen entiteht, während deffon das Platin fich reduoirt. Rs foll nach ihm nur $\frac{x}{8}$ Procent Sauerfoft enthalten. "Iclu lege Ihnen, fulurieb mir Hr. Prof. Dübereiner, etwas von diefem fchwarzen Platin-Suboxyd bei, und zugleich etwas vou Prouft's Knall-Platin, welches frohfarben Ift, und aus I Antheil Platinoxyd und $\frac{x}{3}$ Antheil Ammoniak befteht. Soll letztercs fark detoniren, fo muls es fehr langfam erhitzt werden; beim fchnelien Erbitzen verpuffi os nur fchwach." Gilb.

*) Schweigger's Journal neue Roibe, Bd. 2. 


\section{[ $\quad 195$ ]}

Nun aber beftelt ein Antheil Alkohol aus

$\left.\begin{array}{l}2 \text { Antheilen }=2 \times 11,40 \text { Kohlenftoff } \\ 2-\quad=2 \times 7,50 \text { Sauerfoff } \\ 6-\quad=6 \times 0,95 \text { Wafferfoff }\end{array}\right\}=C^{2} \dot{O}^{\circ} H_{H}$

und ein Antheil Effigfäure aus

$\left.\begin{array}{l}2 \text { Antheilen }=2 \times 11,40 \text { Kohlenfoff } \\ 3-\quad=3 \times \quad 7,50 \text { Saverftoff } \\ 3-\quad=3 \times \quad 0,95 \text { Wafferfoff }\end{array}\right\}=\dot{C}^{2} \dot{O}^{3}$

„Es muls folglich ein Antheil Alkohol 4 Antheile ,Sauerftoff aufnehmen, um in Efligfunre und Waffer "verwaudelt zu werden;" denn es ift

$$
C^{2} O^{2} H^{\kappa}+4 O=C_{C}^{2} \stackrel{B}{H}^{\circ}+3 H O
$$

Und es mufo alfo in diefem Falle I Antheil Alkohol fich in 1 Antheil Elfigfüre und 3 Antheile Wafler rerwandeln.

Um diefes zu prüfen und auf eine Art, bei der fich alles genau mefien liefs, zu beftätigen, hatte ich in die mit $7 \mathrm{Kub}$.Zoll Sauerfloffgas gefïllte Glocko meines kleinen Galometers [Amu. B. 68 S. 8g] 2 Gran

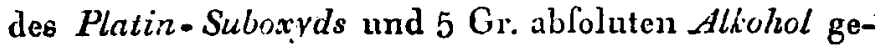
bracht. Zufällig breitete der Alkohol fich nicht fchnell über das Suboxyd aus, und ein Theil des letztern wurde daher glühend. Faft in demfelben Angenblick entflammte fich der Alkohol, wind bildete eine Knalllnft, welche im fulgenden Angenblicke die Glocke mit einem furchtbaren Knall in meiner Hand zermalmto und das fperrende Queckfilber umier zerftrent:. Iclı hätle dabei leiclit verwundet, ja verfümmelt werden nnd die Angen verlieren können, die ich glücklicher Weile als ich die Flamme entf́thn fals fogleicht 


\section{[ 196$]$}

fchlofs, denn ich fülltte an den Augenliedern, fo wis. am ganzen Geficht, heftige Stölso von fortgefchlendertem Queckfilber. Die Augen blieben unverletzt, dic Hant der rechten Hand aber war an mehreren Stellen von Glastrünmern zerfchinitten, and an einenı Finger entftand eine fichtbar wachfende Gefcliwulft, welche aber bald wieder verging. Furclitbar war die Zerftörung in dem kleinen Raume des Experinents, und die Glastrümmer und ein Theil des Queckfilbers waren bis an dio Wande and die Decke meines 30 Fuls langen, $25 \mathrm{Fufs}$ breiten und 15 Fufs hohen Experimentirfaales gefchlendert worden; Umftünde, welche ich anführe, umn andern, welche jenen Verfuch wiederholen wollen, die damit verbundene Gefahr kennen zu lehren. Man mufs dabei höchlft vorfichtig zu Werke gelien, und das Platin-Suboxyd nur in Jichwach mit W'affer befeuchtetem. Zuffande mit dem Alkohol in Berührung bringen, zur gröfsern Sicherheit anch lieber atmofphäifclie Luft als Sauerftoffgas nelimen; es erfolgt dann keine Entzündung.

Noch an dem Tage des unglücklichen Verfuchs brachte ich 100 Gran befenchtetes Platin-Suboxyd mit 100 Gran abfoluten Alkohol unter eine grofse Glocke voll atmofphärifcher Luft, und fchon nach a Stunde fand ficl der Alkohol ganz in Elfigfaure verwandelt. Ift die Glocke graduirt und mit Queckfilber gefperrt; fo kann man ane der Gröfse der Verminderung der Luft (nach vorhergegangener Correction in Beziehung anf die Tenfion des Dampfes der gebildeten Effigfaure), die Menge des Sauerftoffgales, welche von einer gegebenen Quantitat Alkohols verzehrt worden ift, fehr genau berechnen. Nach den Refultaten mch- 


\section{[ $\quad 197$ I}

rerer folcher Verfiche, wird von einem Gran abfoluten Alkohol ftels 1,71 rheinl. Duodecimal-Kubikzoll Sauerftoffgas von derjenigen Dichte, wic fie bei einem Barometerftand von 28 par. Zoll und einer Temperatur von $10^{\circ} \mathrm{R}$. ift, abforbirt, - allo genan fo viel, wie die Rechnung angiebt. Klebt man an die innere Flăche der Wand der Glocke einen kleinen Streifen mit Waffer befencteten Lacknuspapiers, fo fieht man an der anfangenden und zunehmenden Röthung deffelben zugleich das Beginnen und den Fortgang der Effigfure-Bildung, und man kamn fo durch Verfuche allein die Theorie von der Bildung der Effigfäure ans Alkohol in einer einzigen Lehrftunde entwickeln, fo wie auch durch einen Gegen-Verfuch zeigen, dafs der Alkolıol für fich, d. h. ohne einen folchen electrifchen Gegenfatz wie das Platin-Suboxyd ift, kein Sauerftoffgas abforbirt und nicht zu Elfigläure wird ").

Uebrigens orleidet das Platin-Suboxyd bei diefer Metamorphofe des Alkohols keine Veränderung, und man kann daffelbe fofort gebranchen, um neue, vie]leicht unendliche Quantitäten Alkohols zu lüuren; ein Umftand, welcher nicht allein für die rein electri-

*) Wollen Sie diefen Procefs der Effigfaure-Bildung felbot veranlafien, und fich van der angegebnen Gröfse der SauerftoffAbforption überzeugon, fo breiten Sie nur auf Qneckfilbor ein Stiickchen weifses Drackpapier von der Gröfse eines Laubthalers aus, befeuchten es mit etlichen Tropfen Waffer, ftreuen daun etwa I Gran des Platin-Suboxydo darauf, und wenn es etwas Feuchtigkeit aufgofogen hat, tröpfeln Sie I oder 2 Gran abfoluten Alkohol hinzu', und bedecken das Papier fogleich mit einer mit Luft gefüllten Glocke. Nach 24 Stunden zeigt fich Enfigfaure und die augegebue Sauerftof-Verminderung. $D$. 


\section{$\left[\begin{array}{lll}1 & 1 & \end{array}\right]$}

fche Function des genamuten Iraparats fpriclit, fondern anch die Anwendung deffelben zur Darftellung der Effigfüure im Grofsen zulälfig macht.

Anch das anf hydro-chemilchem Wege dargeftellte Schweftel-Platin macht, wenn es einige Tage an der Luft geftanden und fich allo partiell gefänert hat, (demn es zielht fehr begierig Sanerftoff an) den Alkohol pofitiv - electrifch und fïlst ihn in Elfigfanre ïber. Da aber hierbei auch vom Scliwefel-Platin felbft noch Sauerftoffgas verfchluckt wird, fo eignet fich daffelbe nicht gane zu obigem dogmatifch - chemilchen Zweck, wohl aber zur technilchen Anwendung.

Wir kemmen alfo runmehr drei Arten des $\mathrm{Ver}$ brennens des Alkohols, nämlich 1) das dunkele Verbrennen, 2) das glïhende Verbrennen, und 3) das flammende Verbrennen defelben. Beim dumkeln oder ftillen lichtlofen Verbrennen entftehn Effigfünre und Walfer; beim glühenden Verbrennen brenzliche Effigfuture (Lampenfäure), Kohlenfäure und Watler; und beim flammenden Verbrennen Feuer, Kohlen1fiure und Waffer.

Jena d. 15 Januar 1822 .

Was ich Ihnen über das Verhalten des Alkohols, wenn er mit dem Suboxyd des Platins in Berührung fteht, gegen Sauerfoffgas genneldet labe, habe ich oft wiederholt, und jederzeit beftütigt gefunden. Immer nimmt a Antheil Alkohol 4 Antheile Sauerftoffgas in fich auf, und bildet damit 2 Antheil Effigfäure.

Das letztere läfst fich anch daraus beweifen, dafs die erzengte Effiglïure aus vollkommen gefittigtem kohlenfauren Kali oder Natron ein Volumen Kohlenfäure- 


\section{[ $\quad 399$ ]}

gâs entwickelt, welches der haummenge dis abforbirten Squerfiofigales genon gleichili, datier ans a Antheil Aliohol anch \& Antheil Efligfüre gebildet feyn mufs. Drücken wir den Alkolıol durciı die Fornel $c^{2} O^{2} \stackrel{6}{H}$, den Sanerftoff durch das Zeichen $O$ und die Effiglüre durch $\stackrel{2}{C}^{3} H^{3} \mathrm{a}$ aus, fo kann alfo jene merkwärdige Metamorphole des Alkohols durch die Formel $\stackrel{2}{C} \stackrel{6}{H}+{ }_{4} O=\stackrel{2}{C}^{O} \stackrel{3}{H}+3 . H O$ verfinnlicht werden, und man fieht, dafs mit dem 1 Antheil Effigfautre gleichzeitig auch 3 Antheile Wafter entftehen.

Bei diefer Unwandlung des Alkohols in Effigfiure durchdringen fich alfo gleiche Raumtheile Alkoholdampf und Sauerftofigas. Da nun, wie ich zuerft auf dem Wege des Experiments dargethan liabe, bei der Gälrung des Zuckers gleiche Ranmthcile Alkoholdamp $\mathrm{f}$ und Kolhlenfüregas gebildet werden, und in letzteren ein demfelben gleiches Volumen Saucrfoftgas enthalten ift, lo fielıt man, dafs jene Producte der Oxydation des Alkoliols $\left(C^{2} O^{3} H^{3}+5 . H O\right)$ gleich find Zucker $\left(=\stackrel{3}{C} \stackrel{6}{O}^{H}\right)$, welchem 1 Antliejl Kollenftoff entzogen worden, indem $C^{2} O^{3} U^{3}+3 . H O=C^{3} O^{6} \stackrel{\circ}{H}-\mathrm{C}$ ift. Es würde fich dielem zu Folge der Zucker unmittelbar in Efiggfüure und Wafler verwandeln, wenn man ihnı 1 Antheil Kohlenftoff entziehn könnte. Diefes gefclieht, theilweife wenigftens, in der That bei der VerkolJung des Zuckers; und könnten wir dielen Procel's geJiorig, das heilst, lo leiten, dafs ficlt keine Kohlenläure, kein, Kohlen-Wafferftoff und kein empyreumatitehcs Gel bei dem Verkohlen bildete; lo würden die 


\section{[ 200 ]}

Producte der Verkohlung eines Antheils Zucker $=C+\stackrel{2}{C}{ }^{3}{ }_{H}^{3}+3 . H O$ feyn.

9. Chemifche Conftitution und neue Verhaltaifo der Ameifonfăure und der Weinfteinfaure, [Herbft 1821.]

Daffelbe einfache Mittel, welches mir dio chemifche Conftitution der Oxalfurre offenbart hat, [f. S. 208], hat mir nun auch die chemilche Natur der Ameifenfäure bekannt gemacht. Behandelt man natmlich die Ameifenflure oder irgend eine ihrer Verbindungen mit den bafilchen Oxyden, mit etwa dem 10 fachen Gewicht concentrirter Schwefelfaure, welche nicht rauchend feyn mufs) fo zerfallt fie ganz in

$$
\begin{aligned}
& \text { 24,30 Gewichtstheile Waffer und } \\
& 75,70 \quad-\quad \text { Kohlenoydgas. }
\end{aligned}
$$

und man findet durch Berechnung, dafo fie aus

x Raumtheil Wafferdampf und

2 - Kohlenoxydgas

zufammengefetzt jift. Eine Menge von einem Alkali oder einem bafilchen Metalloxyd, in welchem 7,5 Gewichtstheile Sauerftoff enthalten ift, nimmt 34,85 Gew. theile reiner Ameifenfunre auf; und in diefer Menge der Säure find entlıalten

$$
\begin{aligned}
8,45 \text { Gew.Th. Walfor } & =\left\{\begin{array}{l}
0,95 \text { Wafferftoff } \\
7,50 \text { Sauerftof }
\end{array}\right. \\
26,40-\text { Kohlenoxyd } & =\left\{\begin{array}{l}
11,40 \text { Kohlenftoff } \\
15,00 \text { Sauerftoff }
\end{array}\right.
\end{aligned}
$$

Da hierin a Antheil Kohlenftoff und 3 Antheile Sauerftoff vorhanden find, und beide Elemente in diefem Verhaltnifs die Oxalfaure bilden, fo hielt ich 


\section{[ $\begin{array}{ll}201 & \text { ] }\end{array}$}

anfangs die Ameifenfüure für Oxalfunre, welche mit Wafferftoff verbunden fey, oder für $\mathrm{C}^{3} H$. Aber snachherige Verluche über ihr Verhalten gegen $\mathrm{H}_{y p e r-}$ oxyde, fo wie gegen Queckfilber- und Sylber-Oxyd haben mir bewielen, dafs fie eine $\nabla$ erbindung von Kohlenoxyd mit Waffer ift. Behandelt man fie nämlich mit einem der genannten Oxyde in gelinder Warme, fo zerfallt fie in Kohlenfaure und Wafter, während das Oxyd allen Sauerftoff verliert und vollkommen metallifirt wird, und es findet dann fich in dem rückftăndigen Wafler keine Spur von Oxalfäure, dergleichen doch frei werden müfste, wenn die Ameifenlaure eine Verbindung von $\mathrm{C}^{3} H$ wäre.

$\mathrm{Ob}$ die Salze der Ameifenfäure vollkommen entwåtert, d.h. alfo in Verbindungen von bafifchen $\mathrm{Oxy}$ den mit Kohlenoxyd verwandelt werden können, foll durch fernere Verfuche ausgemittelt werden.

Mögen die hier mitgetheilten neuen Thatfachen, nămlich 1) dals die Ameifenfüure von Schiwefelfaure im Kohlenoxydgas und Waffer zerlegt wird, und 2) dafs Silber - und Queckfilber - Oxyd von der AmeiCenfaure vollkommen reducirt werden, - von den Chemikern, oder vielmelr von den chemifchen Phyfikern freundlich aufgenommen, und als ein neuer Beitrag zur Erweiterung unferer Kenntnife von der Zufammenfetzung organifcher Erzeugnifle betrachtet werden.

$$
\text { Jena d. } 15 \text { Jan. } 1822 .
$$

Die kriflallifirte, waferleere Weinfteinfäure ift von mir vor 6 Jahiren zerlegt worden. Ich habe fie damals als $\stackrel{2}{C}^{2} H^{6} \mathrm{O}$ erkannt, und diefos Verhultnifs ih- 


\section{$\left[\begin{array}{lll}202 & 1\end{array}\right.$}

ver Elemente beftimmte mich, fie als einen chemifchen Gegenfatz des Alkolıols, welcher $=\stackrel{2}{C} \stackrel{2}{O H}$ ift, anzufelın. Dals diefe Anficht nicht ungegründet ift, beweift fich dadurch, dafs fie fich gegen eine grofse Menge Vitriolöl in hoher Temperatur aucl ganz lo wie der Alkolıol verhält. Sie wird nümlich von rauchender Schwefelfüure bei einer Temperatur, wo diefe dem Sieden nahe ift, in zwei Antheile Kollenoxydgas und zwei Antheile Wafler umgeftaltet, indel's der Alkohol unter gleichen Umftänden fich in.2 Antheile ölbildendes Gas (den Gegenfaiz des Kohlenoxydgales), und 2 Antheile Wafler umftaltet.

Nun habe ich aber auch gezeigt, dafs die Ameifenfäure in gleiche Antheile Kolllenoxydgas und Walfer zerfillt, wenn fie mit Schwefelfunre belıandelt wird, und man könnte leicht gegen ein fo analoges. Verhalten zweier fo felır verfchiedener Säuren Zweifel oder Verdacht liegen. Diefes analoge Verhalten ift jedosh nur fcheinbar: die Weinfteinfäure wird nämlich erft in hoher, die Ameifenfüure, fo wie auch dis Oxalfäure aber fchon in niedever, Tempcratur von der Schwefelfiure zerfetźt, und mul's fich daher von letzterer in Rückficht ihrer chemilchlıen Conftitution darin unterfcheiden, dafs fie nicht wie diefe aus Kolilenoxyd und Wafer, fondern nur aus den Elementen diefer beiden.Verbindungen, etwa aus 3 Raumtheilen Kohlenlüure und I Raumtheil KolılenWafferftoff zufammengefelzt ift, denn es ift

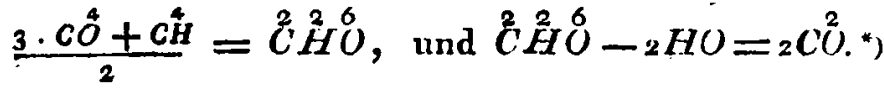

•) Spätor entdeckte Hr. Hofrath Däbereiner die merkwürdige 


\section{$\left[\begin{array}{ll}203 & 0\end{array}\right.$}

3. Ueber die chemifche Conftitution der Gallusfiure;

[im Horiste 1821.]

Ich finde, dafs die Gallusfäure, (fie mag durch Deftillation der Galläpfel, oder durch Belıandlung eines wälferigen Galläpfel-Auszugs mit kohlenlaurem Barit, und nachherige Zerfetzung des gebildeten gallusfauren Barits gewonnen foyn), diefelbe ausgezeichnete Eigenfchaft als die Ameifenfäure hat, die Oxyde des Silbers und des Queckfilbers aus jliren Auflöfungen in Salpeterfäure metallifch niederzufchlagen. Darin aber unterfcheidet fich diefe ihro Wirkung von der der Ameifenlunre wefentlich, dafo fie diefes o/tne Entwickelung von Kohlenfäuregas oder einer andorn elaftifchen Flit'figkeit bewirkt. Sie mul's daher eine trafjerflofffäure leyn, d. h. eine Säure, welche in Hinficht illrev chemifchen Conftitution der Blaulüure ämlicl ift, ( mit der fie auch fonft fo manches im chemifclien Verlaiken Ucbereinftimmende hat); und fie bildet mit den bafifchen Erzmetalloxyden nur aus den Grunde unauflösliche Verbindungen, weil fie den Theil jlıres Valferftoffs, der ihr den Charakter der Acidität giebt, an den Sauerftoff der Oxyde entliifst.

Diejenigen Oxyde, welche ilıren Sauerftoft' fehr feft gebunden entlıalten, wie die Alkalien und die al-

Metamorphofe der Weinfteinfäure in Ameifenfaure (durch Erwarmung tiber fchwarzen Braunftein und Waffer, unter Bildung von Kohlenfiuure und Wafier), welche er in St. 5 des jetz. Jahrg. dief Annalen bekannt gemacht hat, und die von Hrn Gay-Luffac als völlig richtig̨ bentätigt worden if. (St. S. 110.) Gilb. 


\section{[ 204́ ]}

kalifchen Erden, bilden mit der Gallusfüure im Waffer aullösliche Verbindungen, weil fie nicht fähig find, den Wafferftoff derfelben anzuzielıı. Jedoch wird, wenn fic fich in aufgelöften Zuftande befinden, fehr leicht ilirer Siure durch den Sauerftoff der Luft aller Waflerftoff entzogen, und fie lelbft gehen dann in den grün-farbigen Zuftand über, verhalten fich allo beinahe wie die reinen blaufauren Alkalien. Schüttelt man ein in Wafter anfgelöftes Gallusfaures Alkali mit Mangan-Hyperoxyd oder mit Silberoxyd, fo wird die ganze Flüfligkeit in wenig Augenblicken fmaragdgrün, - ein einfaches Mittel, um die Gegenwart der Gallusfärure in einenı mit kohlenfaurem Barit behandelten Pflanzen-Extract $z \mathfrak{u}$ erforfchen.

Berzelius hat die Gallusfure in ihren mit Bleioxyd verbundenen Zuftande analyfirt und fie erkannt als eine Verbindung von

$$
\begin{aligned}
& 3 \text { Antheilen }=3 \times x I, 4 \text { Kohlenftoff } \\
& 3=3 \times 7,5 \text { Sauerfoff } \\
& 3=\quad=3 \times 0,95 \text { Wafferftoff. }
\end{aligned}
$$

Warre diefes ihre wahre Conftitution, fo könnte fie als eine Verbindung von gleichen Antheilen KollIenoxydgas und ölbildendem Gas, (als $1,5 \cdot C^{2} O+1,5 \cdot\left(\stackrel{2}{H}^{2}\right)$ betrachtet werden; aber iclı glaube, dafs diefes Mifchungs-Verhaltnils dem Radical der Gallusfäure angehöre, weil das, was Berzelius für gallusfaures Bleioxyd angelehn hat, nach den obigen Erfahrungen nur dus Refultat der Verbindung des liadicals der Gallusläure mit dem metallifchen Ihadical des Bleioxy ds l'eyn konnte. 


\section{$\left[\begin{array}{lll}205 & 05\end{array}\right.$}

4. Fin neues Verfahren zur Beftimmmng des fïchiometrlfchen

Werthes der Sauren, erlăutert an der Weinfteinfauure,

[den 15 Januar 1822.]

Im zweiten Theile meiner „Mikrochemie“ (in welcher vieles ans Ihren vortreffliclien Annalen entlehnt ift), labe ich ein nenes Verfahren zur Be= ftimmung des ftöcliometrifclien Werths der Sălren befchrieben, von welchem ich glaube, dafs es Ihren Beifall haben werde. Iclı bringe nämlich eine beftimmte kleine Menge der zu prüfenden Siure, in einer mit Queckfilber gefüllten graduirten Glasrölıre, mit einer concentrirten Auflöfung des vollkommen gefätigten kohlenfauren Kalis oder Natrons in Berührung, und beftinme aus der Raum-Menge der Kohlenfïnre, welche entbunden wird, die SättigungsCapacitut der andern Säure, durch Rechnung.

Als ich auf diefe Art 4 Gran kryftallifirter Weins fleinfäure mit einer Auflöfung von kolulenfaurem $\mathrm{Na}$ tron behandelte, erhielt ich als pneumatifches Refultat ein Volumen Kohlenfüuregas, das auf 28 par. Zoll Barometerftand und $10^{\circ} \mathrm{R}$. Temperatur zurückgefülırt, 3,93 rheinl. Duodecimal-Kubikzolle betrug. Da nun $\frac{3.93}{4}=0,9^{825}$, fo fieht man, dals die 4 Gran kryftallifirter Weinfteinfüure eine Menge von Natron fätigten, in welcher 0,9825 rheinl: Kub. Zolle Sauerftoffgas enthalten if *); oder dafs, da 3,93 rheinl. Kub. Zoll

*) Es find nämlich in der Kohlenfäure 4 Antheile Sauerftoff enthalten, daher man nur die Volumen-Menge der entbundnen Kohlenfäure mit $4 \mathrm{zu}$ dividiren braucht, um die Menge des Sauerftoffs zu finden (.. Ann. 1821 St. 3 S. 333.) 


\section{[ 206 ]}

Kolılenfüuregas unter jenem Drucke etc. 2,12 Gran wiegen, 4 Gran kryftallifirter (nach Berzelins mit 1 Antheil Kryltallwaller begabten) Weinfteinfäure ein Aequivalent find für 2,12 Gran Kolnlenfäure. Wenn wir unu den ftöchiometrifchen Werth der Kohlenfäıre durch die 'Lahl 41,4 darfellen, (weil 41,4 Gewtlle derfelben fich mit einer Menge von Kali oder Natron verbinden, in welcher 7,5 Gewthle Sauerftoff enthalten find, um eine neutrale kohlenfaure Verbindung zu bilden), fo mufs die kryflallifirte Weinfteinfüure durch die Zahl 78,12 dargeftellt werden; denn es ift 2,12:4= 41,4:78,12. Ziehen wir von diefer Zahl 8,45, als die Menge des in derfelben enthaltenen $\mathrm{K}_{\mathrm{ry}}-$ ftall-Walfers ab, fo bleibt für die waferleere Weinfteinfäure die Zahl 69,67 , wio ich (chon frülıer gefunden laabe. (Schweigg. Journ. B. 17 S. 06 g.)

\section{Nachfchrift von Gilbert,}

Hrn Döbereiner's pneumatifche Microchemie, Zerlegung der Sauerkleefäure, und Wiederholung des Leidenfroft'fchen Verfuchs betreffeud.

„Zur milro-chemifchen Experimentir-Kunfl, von Dr. J. W. Dübereiner, 2 Theile, Jena 1821, ( 20 gr. beide) " ift der Titel des dem Zweck und der Ausfïlırung naclı empfehlungswerthen, für Anfänger in der Chemie befimmiten, aber anclı für Chemiker recht brauchbaren Werks, auf das Hr. Prof. Döbereiner fich S. 205 bernft. Ein zweiter Titel nennt es: "Źur pueumailichen Chemie", und der Verfaficr felbft auch : "Zur pnenmatifchen Mikrochenue". Der erflé 